\title{
Foreword: Flow numerical simulations in textile technologies
}

\section{Lead Guest Editor: Karel Adamek}

VUTS, A. S., Svarovska 619, 46001 Liberec XI, the Czech Republic

\section{Email address:}

karel.adamek@vuts.cz

\section{To cite this article:}

Karel Adamek. Foreword: Flow Numerical Simulations in Textile Technologies. International Journal of Mechanical Engineering and Applications. Special Issue: Moving Forward to Monitory Democracy: Citizens Engagement in Scrutinizing Election Process in Indonesian 2014 General Election. Vol. 3, No. 1-1, 2015, pp. 1-4. doi: 10.11648/j.ijmea.s.2015030101.11

\section{Abstract \\ The monograph presents the overview of typical numerical flow simulations, solved in VÚTS - Center for Research in Machinery in Liberec (former Research Institute of Textile Machines) in the area of fluid mechanics for various technical applications. First of all in textile technologies, as for instance air jet weaving, spinning, drying, permeability, nonwovens etc. System is completed by auxiliary technologies as for instance air conditioning, exhausting, pneumatic transport etc.}

\section{Personal Profile of Lead Guest Editor}

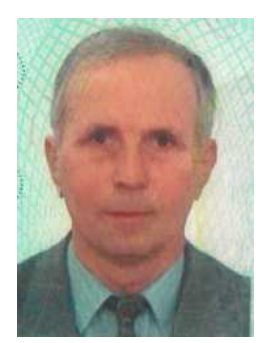

Name: Karel Adamek

\section{Titles and Degrees}

2005

1992

1982

1967

\section{Education}

1974 - 1979 Doctoral study, Fac. of Machinery, Tech. Univ. in Liberec

1974 - 1976 Post gradual courses of university pedagogy, state exam, Tech. Univ. in Liberec

1962 - 1967 Fac. of Machinery, Tech. Univ. in Liberec, spec. Machines designing

\section{Summary of Employment}

2006- ... Research worker at Research Dept. Numerical simulations at VÚTS Liberec, Inc.

1995 - ... $\quad$ Assoc. Prof., later Prof. at Dept. of Power Equipment, Fac. of Machinery, Tech. Univ. in Liberec (part time) 2002 - 2006 Head of the Dept. of Flow Numer. Simul. at VÚTS Liberec 
1984 - 2002 Research worker at Research dept. of weaving engineering at Elitex KVÚ Liberec, later VÚTS Liberec Inc.

1981 - 1984 Looms designer - Elitex k. p. Chrastava

1975 - 1980 Technical lectures at Energy Institute for State Energy Inspection

1970 - 1980 Lecturer at dept. of power equipment, Fac. of Machinery, Tech. Univ. in Liberec

1967 - 1970 Designer of truck motors Škoda - LIAZ n.p. Jablonec n. N., military service

\section{Editorial Group}

\section{Lead Guest Editor:}

Karel Adamek, VUTS Liberec, Czech Rep., Dept. of flow numerical simulatins, karel.adamek@vuts.cz

Guest Editors:

Jan Kolar, VUTS Liberec, Czech Rep., Dept. od flow numerical simulations, jan.kolar@vuts.cz

Pavel Peukert, VUTS Liberec, Czech Rep., Dept. of flow numerical simulations, pavel.peukert@ vuts.cz

Stanislav Juklíček, VÚTS Liberec, Czech Rep., Dept. of informatics, stanislav.juklicek@vuts.cz

\section{Acknowledgments}

This overview summary publication was created under the support of the Czech grant No. LO 1213 - Excellent machinery research.

\section{Content}

Foreword

Karel Adamek

Main weaving nozzles (ejectors)

Karel Adamek, Jan Kolar,

Jaroslav Pelant, Jan Šimák, Simone Gramsch

Relay nozzles and weaving reed

Karel Adamek, Petr Karel, Jan Kolar, Václav Kopecký,

Slavomír Jirkì, Jaroslav Pelant

Air conditioning

Karel Adamek, Jan Kolar, Pavel Peukert, Marek Kašpar

Wall flows

Karel Adamek, Dušan Čejka, Zdeněk Mečl, Jaroslav Pelant

Exhausting, pneumatic transport

Karel Adamek, Jan Kolar, Pavel Peukert, Martin Pustka, Petr Pi̊lpán,

Aleš Prǔšek, Radim Foldyna,

Air permeability, drying, blowing

Karel Adamek, Jaroslav Fábera, Milan Stejskal,

Antonín Havelka, Stanislav Franc

Flow numerical simulation in various technologies

Karel Adamek, Jan Kolar, Pavel Peukert, Jozef Kaniok, Jiři Štorek,

Marcel Havrda, Jaroslav Pelant, Martin Kyncl,

Conclusion

Karel Adamek 


\section{Foreword}

VÚTS, former Research Institute of Textile Machines was established in Liberec in the year 1951 and it is permanently widely engaged in the research of textile machines. Let us to remind two examples, only:

The first air jet loom prepared for serial, it means the mass production (as the maximum of 5000 pieces per year), was designed in our institute.

The first principle and function model of the open end spinning was designed in our institute, too, and later transferred to the producer.

Both systems, based on air flows, are today used worldwide as standard, modern, reliable, efficient and powerful technologies.

Our small department of numerical flow simulations is engaged in many branches of industry now.

Using of air flow arising either by expansion of compressed air or driven by a fan is very spread in many branches of industry. From the physical principle, it is wellknown that energy consumption for air compression is an important item in the total energy balance of operated equipment. Endeavour of its better efficiency is connected with the research of conversion of internal energy compressed air into kinetic energy of air flow. Such conversion is the main operation phenomenon for different kinds of technical equipment. The following text describes some results of air flow research made by numerical modeling, at first in principal parts of air jet weaving system; further of other textiles technologies and some examples from others branches of processing technologies, too.

In connection with the development of calculating methods of finite elements (or volumes) in fluid mechanics and that of computers with an adequate output, too, it is possible to execute numerical modeling of flows now, as a complement or replacing of former experiments. It is clear that every model is an approach to the reality, only, and that it is necessary to verify such results of numerical modeling by experiments or by real operation trials.

In Research Institute for Textile Machines in Liberec/CZ (VÚTS), there was has been used a program [1] since 1994 for numerical modeling of two-dimensional (2D) compressible inviscid flows, based on a numerical solution of Euler's equations system, converted from a modeling flow of water steam. Its application is fully competent for a modeling of quick air flow processes in the branch of air jet looms, as for instance expansion and flow of compressed air in weaving nozzle because the compressibility influence is here more important in comparison with viscous friction. In many solved cases, it was demonstrated that it is often possible to use this program to obtain quickly and simply some qualitative knowledge about the systems with expressively slower flows, too, where it should be more correct to use any procedure considering flow viscosity.

Of course, the solution of axis-symmetrical cases converted into a solution of flat models is not fully correct, too. It is necessary to say that using of results is possible as qualitative ones, only. But as the first imagination for a designer and help for his next designing actions, the influence of this simple method is very positive.

Since 1999 the program [2] has been used, too, for numerical modeling of three-dimensional (3D) viscous compressible flows. The program was developed in Aeronautical Research and Test Institute in Prague/CZ (VZLÚ) for special cases when simple modeling is not adequate. It is based on the numerical solution of NavierStokes' equations system. Its application gives the solutions closer to the reality, by the reason that it contains simultaneous influence of compressibility and viscosity of flowing medium. But the extent of the solved models is so large that their application was limited by output of at the time existing personal computers PC (level of the year 2000). That is why, it was necessary to do expressive simplifications of complicated model geometry so that the model more exact with respect to the thermodynamic and physical qualities of medium becomes less exact with respect to its geometry.

As an option, it was used the program [3] developed in VZLÚ, too, for solutions of compressible viscous flow in axis-symmetrical channels, as the checking method for some cases, initially solved as flat (2D) cases. Using the symmetry condition, the extent of spatial (3D) model is similar to flat (2D) one and time of solution is substantially decreased. Generally, we can state that in the images of the flow field of the cases solved by both methods there are some differences, but the global character of the received flows is quite similar.

As time goes on, the commercial program was used for numerical flow simulations, together with the development of in-house procedures.

Procedures of solution are similar as follows: As to the computational mesh density, it should be the highest to get a maximum detailed flow field, but simultaneously the solution time is increasing. From the thermodynamic conditions, defined usually as a pressure ratio between inlet and outlet with corresponding temperatures, the flow /velocity in the modeled area is arising. The presented flow fields (usually as velocity or Mach number isolines) give the best image about the flow qualities in the solved or observed area.

The scales used for individual presented flow fields are different to get an optimum image about it. Due to place saving, the scales are always missing. In general, the smallest value is blue/violet and the greatest one is red, identically with the wave lengths of color components of white light.

From our experience, it is possible to state that for technical practice (design, technology, etc.) it is not necessary to get a mathematically exact solution of the problem. To understand the problem and to determine the next process of the solution it is usually sufficient to determine the influence of a certain parameter in a modeled system on their operation, efficiency, reliability, etc.

The publication contains several papers focused on important or typical themes. Regarding the extent of the 
submitted texts, some main received results are shortly descripted, only, in the form of flow field images completed by their short discussion, eventually, it is indicated the reason and manner of individual application. A more detailed analysis of results is contained in several research reports and published contributions, some of them are quoted in references at the end of each paper.

From the presented theoretical results and from their technical applications results that the numerical simulations of flow and those of heat exchange are very useful instruments when solving tasks from the technical practice, first of all, in two basic areas:

a) Numerical model is able to confirm or refuse with sufficient accuracy a new hypothesis of the solution for a new technical problem.

b) Numerical model enables to display the behavior of an existing monitored system, to indicate a possible reason of its wrong functioning and to indicate the procedure of its remedy.
In both cases, it is not necessary to produce and test time and money demanding testing equipment, to adapt them and to test them again. Numerical simulations give the right direction of the solution; the necessary experimental verification is carried out on the optimal model, only, which, on the basis of performed simulation could be probably the best for realization.

Next papers present several typical areas, where the numerical simulations were used and their results were applied. Of course, it is not any complete overview of problems.

\section{References}

[1] M. Šejna: Software "CoChem Flow", PC Progress Praha, 1994

[2] J. Pelant: Software "3D weaving channel", VZLÚ Praha, 1999

[3] Pelant, J.: Software "OsoSym", VZLÚ Praha, 2000 